\title{
THE EMERGENCE OF SUBSURFACE SPIN-WAVES IN FCC (110) MAGNETIC FILMS
}

\author{
S. MAmica ${ }^{a}$, H. PUSzKarski ${ }^{b *}$ AND J.C.S. LÉVY ${ }^{b}$ \\ ${ }^{a}$ Surface Physics Division, Faculty of Physics, Adam Mickiewicz University \\ Umultowska 85, 61-614 Poznań, Poland \\ ${ }^{b}$ Laboratoire de Physique Théorique de la Matière Condensée \\ Université Paris 7-Denis Diderot, 2 Place Jussieu, 75221 Paris Cedex 05, France
}

We show that the emergence of subsurface branches in the spin-wave spectra of thin films is due to the deficiency of interactions with next-nearest-neighbours, the position vectors of which are oblique to the film surface.

PACS numbers: 75.70.-i, 75.40.Gb, 75.30.Ds

1. Recent years have witnessed a revival of interest in the interaction of next-nearest-neighbours (NNN) in many different fields of research (see our recent papers $[1,2]$ and references therein). Here, we shall consider the model of a ferromagnetic $\mathrm{fcc}_{\mathrm{c}}(110)$ thin film involving both nearest-neighbour (NN) and next-nearest-neighbour (NNN) exchange interactions. We perform our calculations within the framework of the Heisenberg localized spin model, assuming an exchange (NN and NNN) and Zeeman Hamiltonian in a standard form

$$
\widehat{\mathcal{H}}=-2 J_{1} \sum_{\left(l \boldsymbol{j} ; l^{\prime} \boldsymbol{j}^{\prime}\right)} \widehat{\boldsymbol{S}}_{l \boldsymbol{j}} \cdot \widehat{\boldsymbol{S}}_{l^{\prime} \boldsymbol{j}^{\prime}}-2 J_{2} \sum_{\left(\boldsymbol{l} ; l^{\prime} \boldsymbol{j}^{\prime}\right)}^{\mathrm{N}} \widehat{\boldsymbol{S}}_{\boldsymbol{l} \boldsymbol{j}} \cdot \widehat{\boldsymbol{S}}_{l^{\prime} \boldsymbol{j}^{\prime}}-g \mu_{\beta} \sum_{l \boldsymbol{j}} \boldsymbol{H}^{\mathrm{eff}} \cdot \widehat{\boldsymbol{S}}_{l \boldsymbol{j}},
$$

where $\boldsymbol{H}^{\text {eff }}$ comprises both external and demagnetizing fields. The summations extend over different pairs of neighbouring spins, respectively, $\sum$ stands for the summation over NN and $\sum^{N}$ over NNN. Moreover, $l j$ defines the position of a given spin, with $l$ labelling the atomic layer (parallel to the surface) and $j$ is a two-dimensional vector defining the position of the spin in the $l$-th layer. The NN and NNN neighbours of a given site of the layer $l$ lie in 5 layers, which we shall label as follows: $l^{\prime}=l ; l \pm 1 ; l \pm 2$; the NN and NNN distributions of the projections of the neighbours of a given site onto the plane of the surface are shown in Fig. 1a. We diagonalize the Hamiltonian (1) by applying the procedure described in our former paper [3]; when performing the respective transformations of spin operators we introduce a two-dimensional vector $k_{\|} \equiv\left[k_{1}, k_{2}\right]$ defined in the first Brillouin

\footnotetext{
*corresponding author: e-mail: henpusz@main.amu.edu.pl
} 
(a)

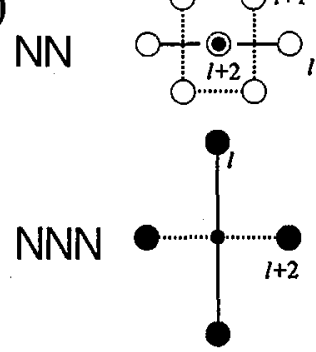

(b)

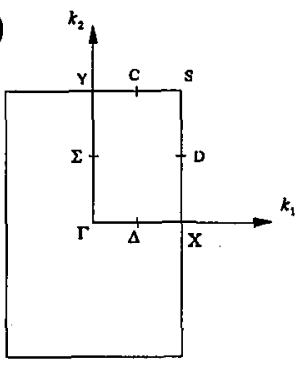

Fig. 1. (a) The dispositions of NN and NNN neighbours and (b) two-dimensional Brillouin zone for fcc (110) surface orientation.

zone of the planar reciprocal lattice (in the film plane). The first Brillouin zone for the surface cut considered is shown in Fig. 1b with points of high symmetry marked on it.

2. The diagonalisation of the Hamiltonian (1) is equivalent to that of the following five-diagonal matrix of the rank $L$

$$
\left(\begin{array}{ccccccc}
R-a_{0}, & C, & D & & & & \\
C, & R-a_{1}, & C, & D & & & \\
D, & C, & R, & C, & D & & \\
& \ddots & \ddots & \ddots & \ddots & \ddots & \\
& & D, & C, & R, & C, & D \\
& & & D, & C, & R-a_{1}, & C \\
& & & & D, & C, & R-a_{0}
\end{array}\right) .
$$

Its elements are defined as follows:

$$
\begin{aligned}
& R=4 S\left[J_{1}\left(6-\cos k_{2}\right)+J_{2}\left(3-\cos k_{1}\right)\right]+g \mu_{\beta}\left(H^{\mathrm{eff}} \cdot \gamma\right), \\
& C=-8 S J_{1} \cos \frac{1}{2} k_{1} \cos \frac{1}{2} k_{2}, \quad D=-2 S\left(J_{1}+2 J_{2} \cos k_{2}\right), \\
& a_{0}=2 S\left(5 J_{1}+2 J_{2}\right), \quad a_{1}=2 S\left(J_{1}+2 J_{2}\right) .
\end{aligned}
$$

With the formulae of Eqs. (3) we get the following dispersion relation:

$$
\begin{gathered}
E\left(k_{\|}, k_{\perp}\right)-g \mu_{\beta}\left(\boldsymbol{H}^{\mathrm{eff}} \cdot \gamma\right)=4 S J_{1}\left(6-\cos k_{2}-4 \cos \frac{1}{2} k_{1} \cos \frac{1}{2} k_{2} \cos k_{\perp}\right. \\
\left.\quad-\cos 2 k_{\perp}\right)+4 S J_{2}\left(3-\cos k_{1}-2 \cos k_{2} \cos 2 k_{\perp}\right),
\end{gathered}
$$

where $k_{\perp}$ is the wave vector component perpendicular to the surface of the film. When determining the permitted branches of the spin wave energy spectra, since we have no knowledge of the quantized values of $k_{\perp}$, we have to rely on the diagonalization of the matrix (2) numerically by the use of an adequate procedure. Our results are given in Fig. 2a, b consisting of energy spectra determined numerically for different values of the exchange integrals $J_{1}$ and $J_{2}$. The reduced energy $\left[E\left(k_{\|}, k_{\perp}\right)-g \mu_{\beta}\left(\boldsymbol{H}^{\text {eff }} \cdot \gamma\right)\right]$ is expressed in units of $2 S J_{1}$; the thin film is 

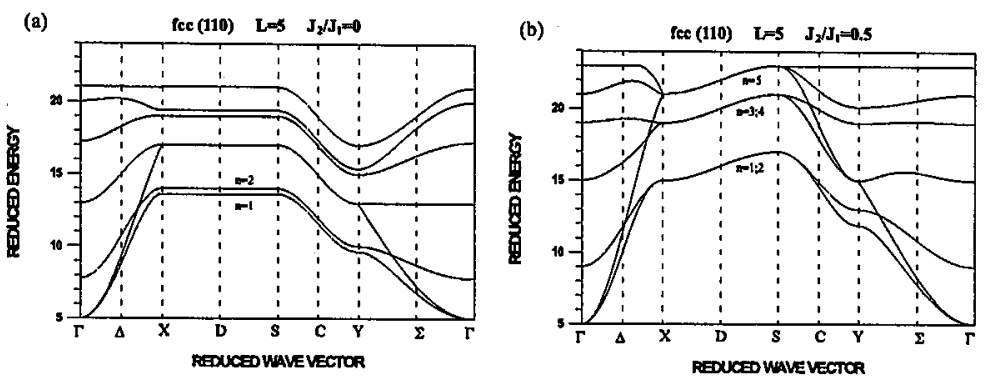

Fig. 2. The reduced spin-wave energy spectra (see Eq. (4)) for a thin film consisting of five layers $(L=5)$ vs. the in-plane vector $k_{\|}$varying along the high symmetry path of Fig. $1 \mathrm{~b}$ for indicated values of the ratio $J_{2} / J_{1}$.
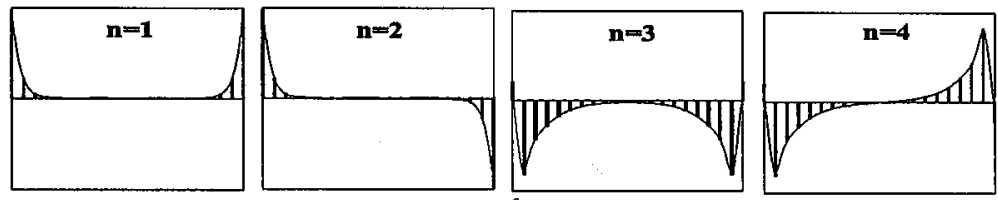

Fig. 3. The formation of surface/subsurface localization of the energetically lowest modes $n=1,3$ (symmetric) and $n=2,4$ (antisymmetric) for $k_{\|}$corresponding to a point in between points $\Delta$ and $X$, see Fig. $2 \mathrm{~b}$.

assumed to consist of five atomic planes $(L=5)$ - a particular value of $L$ chosen to provide good readability for the energy branches. The reduced energy spectra are determined for the surface cut under consideration along the path connecting points of high symmetry of the Brillouin zone, as shown in Fig. 1b (the dense lines mark the boundaries of the band). For the situation when $J_{2}=0$ and $J_{1} \neq 0$ (Fig. 2a), we get a spectrum consisting of 5 branches; the two lowest (surface) states descend below the band in the vicinity of the point $\Delta$ and stay there until we cross a point close to $\Sigma$. Thus, we are justified in attributing the arisal of the two surface branches to NN interactions (see also Fig. 1a). Let us now consider the case of $J_{2} / J_{1}=0.5$ (Fig. $2 \mathrm{~b}$ ); the spectrum obtained exhibits two essential aspects: firstly, in the $X S$ region the bulk band collapses, and secondly, the four lowest states now descend below the band. We readily explain the "band collapse" experienced by the spectrum in the region $X S$ : for the segment $X S$ the coordinate $k_{1}=\pi$ and (see Eq. (3)) the factor $C$ in the matrix (2) vanishes identically. Accordingly, in Fig. $2 \mathrm{~b}$, we obtain in the region $X S$ but three energy levels: the one corresponding to the "bulk" excitation, and the other (lower lying) corresponding to twice degenerate "surface" and "subsurface" excitations. To justify this statement we show in Fig. 3 the profiles of the symmetric modes $n=1,3$ and of the antisymmetric ones $n=2,4$ depicted in the particular point of the Brillouin zone, namely a point in between points $\Delta$ and $X$. We see that in fact the modes $n=1,2$ are of the surface type, while the remaining two $(n=3,4)$ are the modes of the subsurface nature.

3. To grasp the physical implications of the above, let us go back to Fig. 1a and analyse the distributions of the NN and NNN neighbours. The NN neighbours 
lie in the $l$-th plane, are perpendicular thereto (one neighbour belonging to the plane $(l \pm 2)$ ) and lie obliquely to it (four neighbours from the plane $(l \pm 1)$ ); the NNN neighbours lie in the $l$-th plane (two neighbours) and in the $(l \pm 2)$-th plane (two neighbours in the "oblique" configuration). Hence, we can draw the following conclusion: the emergence of surface/subsurface branches in the spectra of thin films is due to the deficiency of interactions with neighbours the position vectors of which are oblique to the film surface, whereas the rupture of bonding with neighbours lying "perpendicularly" above the surface does not favour the emergence of surface/subsurface states.

The present work was supported by the grant No. 2 P03B 07916 from the Committee for Scientific Research and in part by the French-Polish cooperation program POLONIUM.

\section{References}

[1] S. Mamica, R. Józefowicz, H. Puszkarski, Acta Phys. Pol. A 94, 79 (1998).

[2] H. Puszkarski, J.C.S. Lévy, S. Mamica, Phys. Lett. A 246, 347 (1998).

[3] H. Puszkarski, Prog. Surf. Sci. 9, 191 (1979). 